DRAFT VERSION FEBRUARY 27, 2013

Preprint typeset using LTEX style emulateapj v. 5/2/11

\title{
A REDLINE STARBURST: CO(2-1) OBSERVATIONS OF AN EDDINGTON-LIMITED GALAXY REVEAL STAR FORMATION AT ITS MOST EXTREME
}

\author{
J. E. Geach ${ }^{1}$, R. C. Hickox ${ }^{2}$, A. M. Diamond-Stanic ${ }^{3}$, M. Krips ${ }^{4}$, J. Moustakas ${ }^{5}$, \\ C. A. Tremonti ${ }^{6}$, A. L. CoIL ${ }^{3}$, P. H. Sell ${ }^{6}$, G. H. Rudnick ${ }^{7}$ \\ Draft version February 27, 2013
}

\begin{abstract}
We report observations of the CO(2-1) emission of SDSS J1506+54, a compact $\left(r_{\mathrm{e}} \approx 135 \mathrm{pc}\right)$ starburst galaxy at $z=0.6$. SDSS J1506 +54 appears to be forming stars close to the limit allowed by stellar radiation pressure feedback models: the measured $L_{\mathrm{IR}} / L_{\mathrm{CO}}^{\prime} \approx 1500$ is one of the highest measured for any galaxy. With its compact optical morphology but extended low surface brightness envelope, post-starburst spectral features, high infrared luminosity $\left(L_{\mathrm{IR}}>10^{12.5} L_{\odot}\right)$, low gas fraction $\left(M_{\mathrm{H}_{2}} / M_{\star} \approx 15 \%\right)$, and short gas depletion time (tens of Myr), we speculate that this is a feedback-limited central starburst episode at the conclusion of a major merger. Taken as such, SDSS J1504+54 epitomizes the brief closing stage of a classic model of galaxy growth: we are witnessing a key component of spheroid formation during what we term a 'redline' starburst.
\end{abstract}

Subject headings: galaxies: starburst, evolution

\section{INTRODUCTION}

Theoretical studies suggest that the majority of the stellar mass in the central regions of massive spheroids forms in situ in powerful, compact starbursts (e.g. Narayanan et al. 2010). In these systems, radiative feedback from stars is expected to produce an upper limit on the star formation rate density $\left(\dot{\Sigma}_{\star}\right)$ by limiting the density of the star-forming gas ( $\Sigma_{\text {gas }}$, Thompson, Quataert \& Murray 2005; Murray, Quataert \& Thompson 2005). One explanation for the small range in central stellar surface densities observed in the cores of local spheroids is that they formed in compact starbursts where the bulk of the gas reservoir formed stars at the Eddington limit (Hopkins et al. 2010), however observations have only just begun to probe the most extreme $\dot{\Sigma}_{\star}-\Sigma_{\text {gas }}$ in distant powerful starbursts that are the antecedents of massive galaxies today.

Diamond-Stanic et al. (2012, DS12) reported the discovery, with the Wide-field Infrared Survey Explorer (WISE), of a population of moderate-redshift $(z \approx 0.5)$ galaxies that appear to be among the highest density starbursts yet observed. Hubble Space Telescope (HST) imaging reveals that these systems are remarkably compact in the optical bands, with $r_{e} \sim 100 \mathrm{pc}$ (DS12, P. H. Sell et al. 2013 in preparation), while the optical spectra and UV-IR spectral energy distributions imply high star formation rates (SFRs) approaching $10^{3} M_{\odot} \mathrm{yr}^{-1}$ (and thus very high $\dot{\Sigma}_{\star}$ ), with little or no contribution from an active galactic nucleus (AGN). The galaxies also exhibit extremely blueshifted $\left(1000 \mathrm{~km} \mathrm{~s}^{-1}\right)$ Mg II $\lambda \lambda 2796,2803$ interstellar absorption lines (Tremonti et

\footnotetext{
${ }^{1}$ Department of Physics, McGill University, Montréal, Québec, Canada. jimgeach@physics.mcgill.ca

${ }^{2}$ Department of Physics and Astronomy, Dartmouth College, Hanover, NH 03755, USA

${ }^{3}$ Center for Astrophysics and Space Sciences, University of California, San Diego, La Jolla, CA 92093, USA

${ }^{4}$ Institut de Radioastronomie Millimétrique, 300 rue de la Piscine F38406 Saint Martin d'Hères, France

5 Department of Physics and Astronomy, Siena College, 515 Loudon Road, Loudonville, NY 12211

${ }^{6}$ Department of Astronomy, University of Wisconsin-Madison, Madison, WI 53706, USA

${ }^{7}$ Department of Physics and Astronomy, University of Kansas, Lawrence, KS 66045, USA
}

al. 2007) indicative of powerful feedback.

In order to test if these compact galaxies represent the formation of a massive stellar bulge via a feedback limited starburst, we require a measurement of the cold gas reservoir, since Eddington limited star formation places a theoretical upper limit on $\dot{\Sigma}_{\star} / \Sigma_{\text {gas }}$ for the actively star-forming gas. In this Letter we present new observations at $2 \mathrm{~mm}$ of the most extreme galaxy in the DS12 sample with the Institut de Radioastronomie Millimétrique (IRAM) Plateau de Bure Interferometer (PdBI). We measure the $\mathrm{CO}(2-1)$ molecular emission line and derive the gas properties of this remarkable system to test the picture of Eddington limited star formation in a compact starburst galaxy.

\section{SDSS J150636.30+540220.9}

SDSS J150636.30+540220.9 at $z=0.608$ (hereafter 'SDSS J1506+54') was targeted because it is the most extreme system in the DS12 sample, in terms of its star formation rate density. The effective radius measured in HST WFC3 F814W (rest-frame $V$-band) imaging is $r_{e} \approx 135 \mathrm{pc}$ (Figure 1 ), and even the most conservative estimates for the SFR result in densities of $\dot{\Sigma}_{\star} \approx 3000 M_{\odot} \mathrm{yr}^{-1} \mathrm{kpc}^{-2}$. Fitting to the WISE 12 and $22 \mu \mathrm{m}$ photometry, the integrated $8-1000 \mu \mathrm{m}$ luminosity of SDSS J1506+54 is estimated to be in the range $\log \left(L_{\mathrm{IR}} / L_{\odot}\right)=12.6-13.1$, assuming a representative range of spectral energy distributions appropriate for star-forming galaxies (Chary \& Elbaz 2001, Dale \& Helou 2002, Rieke et al. 2009). The dispersion in rest-frame $24 \mu \mathrm{m}$ luminosity using this range of templates is just $0.1 \mathrm{dex}$. A complementary estimate of $L_{\mathrm{IR}}$ is therefore made using $L_{24}$ using the empirical correlation between $L_{24}-L_{8-1000}$ found by Rieke et al. (2008); we find $\log \left(L_{\mathrm{IR}} / L_{\odot}\right)=12.78 \pm 0.06$, consistent with the estimate above. The SFR of SDSS J1506+54 is estimated to be in the range $340-1400 M_{\odot} \mathrm{yr}^{-1}$ assuming the calibration of Kennicutt (1998), scaled to a Chabrier initial mass function. This is in good agreement with the SFR estimated from stellar population fits to the UV-optical-IR photometry (DS12).

The stellar mass is estimated from stellar population template fits to the $0.1-3 \mu \mathrm{m}$ photometry, with $\log _{10}\left(M_{\star} / M_{\odot}\right)=$ $11.12 \pm 0.07$ (assuming a $0.1-100 M_{\odot}$ Chabrier initial mass 

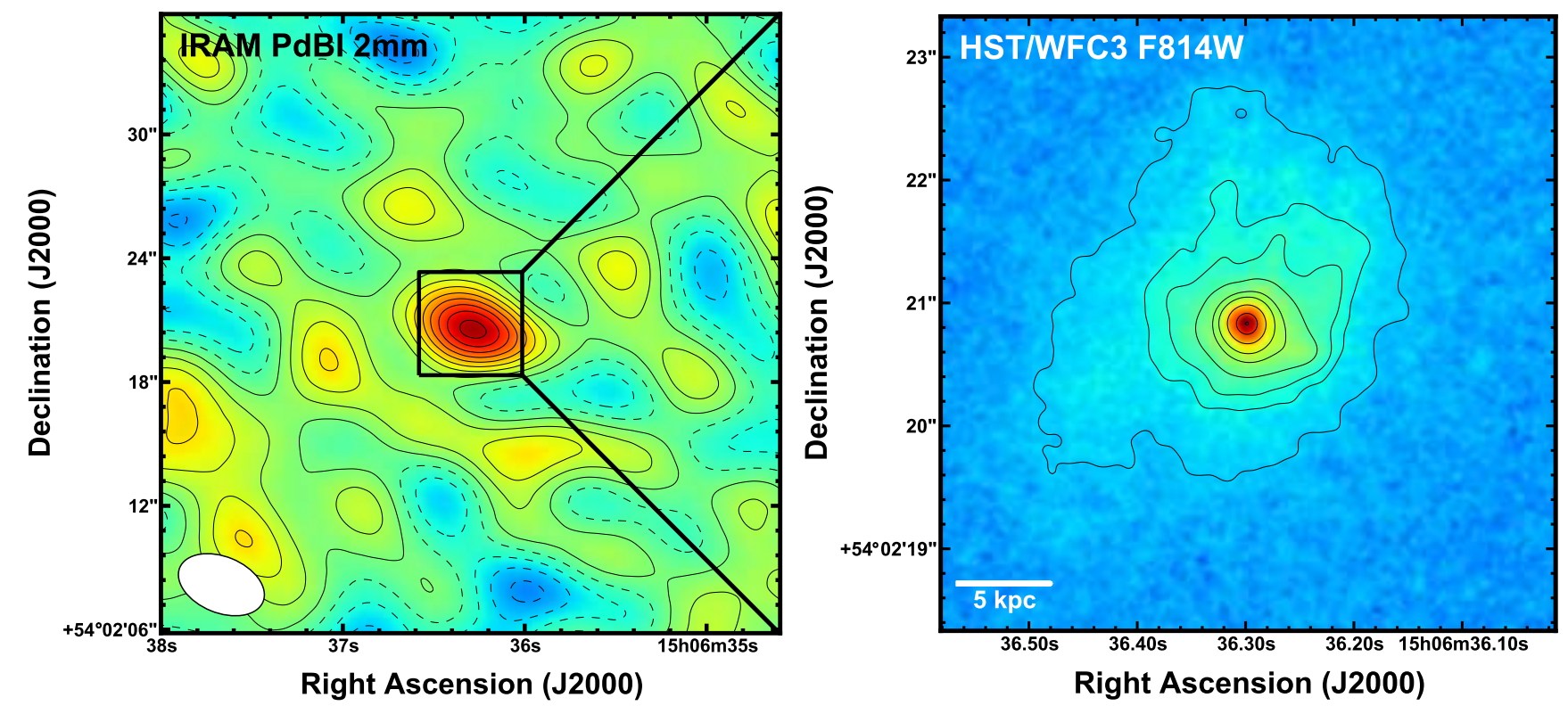

Figure 1. (left) Velocity integrated $\left(\Delta V=360 \mathrm{~km} \mathrm{~s}^{-1}\right)$ IRAM map of the CO (2-1) emission of SDSS J1506+54. The white ellipse shows the $4.4^{\prime \prime} \times 2.7^{\prime \prime}$ beam, and contours are at levels of $0.33 \mathrm{mJy}$ (equivalent to $1 \sigma$; negative values are shown as dashed contours), (right) Zoom-in of $5^{\prime \prime} \times 5^{\prime \prime}$ box (left) showing the HST/WFC3 F814W (rest-frame $V$-band) image. The light is dominated by the compact core, $r_{e}=135 \mathrm{pc}$ (DS12), but fainter, diffuse optical emission is also visible over scales of several kpc indicating a low surface brightness (LSB) envelope of UV emission (contours are shown as a guide). More extensive LSB emission beyond this field of view is also evident, at a level of $\mu_{\mathrm{F} 814 \mathrm{~W}} \approx 25 \mathrm{mag} \mathrm{arcsec}^{-2}$, including a potential tidal tail feature (P. H. Sell et al. in prep). This points to a merger origin for SDSS J1506+54 (§6).

function, see Moustakas et al. 2013). Finally, the outflow velocity of the galactic wind is traced by the interstellar medium $\operatorname{Mg}$ II $\lambda \lambda 2796,2803$ absorption lines, which indicate a maximum $v_{\mathrm{abs}} \approx-1210 \mathrm{~km} \mathrm{~s}^{-1}$ (Tremonti et al. 2007, DS12).

\subsection{Comments on possible AGN contribution}

The source does not appear to be dominated by an AGN. Although SDSS J1506+54 has the most luminous [O III $] \lambda 5007$ and [Ne V] $\lambda 3426$ emission of the DS12 sample $\left(\log \left(L_{[\mathrm{O} \mathrm{III}]} / \mathrm{erg} \mathrm{s}^{-1}\right)=42.1\right.$ and $\log \left(L_{[\mathrm{NeV}]} / \mathrm{erg} \mathrm{s}^{-1}\right)=$ 41.1 ), only 4 counts were detected with Chandra in the 2 $10 \mathrm{keV} X$-ray band, corresponding to a luminosity of $L_{X}=$ $2.9_{-2.9}^{+4.4} \times 10^{42} \mathrm{erg} \mathrm{s}^{-1}$. Assuming typical ratios between $\mathrm{X}$ ray, [O III] $\lambda 5007$ and [ $\mathrm{Ne} \mathrm{V}] \lambda 3426$ luminosities, and accounting for possible small $\sim 30 \%$ dust attenuation of the narrowline region based on the reddening derived from our fit to the SED (DS12), the Chandra limits imply an X-ray absorption factor of $\approx 10-20$ for a buried AGN (DS12, Heckman et al. 2005, Gilli et al. 2010). However, as DS12 argue, even if the $\mathrm{X}$-ray attenuation factor was as high as 100 , the AGN would contribute less than half of the observed $22 \mu \mathrm{m}$ luminosity (Diamond-Stanic et al. 2009, Gandhi et al. 2009). Thus, star formation is likely to be the dominant power source of the bolometric emission of SDSS J1506+54.

\section{IRAM PLATEAU DE BURE 2 MM OBSERVATIONS}

Observations were conducted with IRAM PdBI over JuneJuly 2012 in configuration D, using 5 antennas and the WideX correlator. We targeted the $\mathrm{CO}(2-1) 230.54 \mathrm{GHz}$ rotational transition at $\nu_{\mathrm{obs}}=143.37 \mathrm{GHz}(2 \mathrm{~mm}$ band) in the direction of SDSS J1506+5402. The total (on source) integration time was 6.2 hours (after flagging; scans on-source were discarded for which the phases deviated by more than 45 degrees from the solution), during which time the system temperature ranged between $T_{\text {sys }}=100-400 \mathrm{~K}\left(\left\langle T_{\text {sys }}\right\rangle \approx 200-250 \mathrm{~K}\right)$ for precipitable water vapour in the range pwv $=4-12 \mathrm{~mm}$.
Bandpass calibrators were the sources 3C84, 2200+420 or $3 \mathrm{C} 273$, gain calibration was performed with the sources $1418+546$ and J1604+572 and the source MWC349 was used for flux calibration. The flux calibration accuracy is $\sim 5-10 \%$ at $2 \mathrm{~mm}$. Data were calibrated, mapped and analyzed using GILDAS (Guilloteau \& Lucas 2000).

\section{RESULTS}

Figure 1 shows the velocity integrated $\mathrm{CO}(2-1)$ map of SDSS J1506+54 and Figure 2 presents the $2 \mathrm{~mm}$ spectrum around $\nu_{\mathrm{obs}}=143.37 \mathrm{GHz}$. The $\mathrm{CO}(2-1)$ line is detected with high confidence at $>8 \sigma$ in the integrated map. The integrated line flux is $S \Delta V=0.97 \pm 0.19 \mathrm{Jy} \mathrm{km} \mathrm{s}^{-1}$, corresponding to a luminosity of $L_{\mathrm{CO}(2-1)}^{\prime}=(4.8 \pm 0.9) \times$ $10^{9} \mathrm{~K} \mathrm{~km} \mathrm{~s}^{-1} \mathrm{pc}^{2}$. Uncertainties are conservative; they are estimated via a bootstrap analysis, where we generate a series of realizations of the spectrum, adding noise to each $40 \mathrm{~km} \mathrm{~s}^{-1}$ channel, selected from a Gaussian distribution with $\sigma=1.1 \mathrm{mJy}$. The standard deviation of the ensemble of integrated spectra are taken to be the $1 \sigma$ error of the measured line flux.

The line profile is adequately fit by a single Gaussian profile $\left(\chi^{2} / \nu=1.1\right)$, with a velocity width FWHM $=283 \pm$ $31 \mathrm{~km} \mathrm{~s}^{-1}$ (Fig. 2). Assuming the average inclination angle $i=30^{\circ}$, the true width could be as large as $\sim 560 \mathrm{~km} \mathrm{~s}^{-1}$. Galaxy-integrated $\mathrm{CO}$ spectra often exhibit double peaked line profiles, indicative of a rotating disc or ring (e.g. Downes \& Solomon 1998). There are some hints of this in the current spectrum, and so we also fit a double-Gaussian profile (fixing the individual line peaks and individual dispersions to be equal). The fit is formally $\chi^{2} / \nu=0.9$, not significantly improved over the single component fit. The peak-to-peak velocity is $\Delta V=(147 \pm 12) \mathrm{km} \mathrm{s}^{-1}$, and the line dispersion, which includes the 1-dimensional turbulent velocity dispersion of the gas, is $\sigma=(49 \pm 6) \mathrm{km} \mathrm{s}^{-1}$.

At first glance, it seems puzzling that the line width is not 


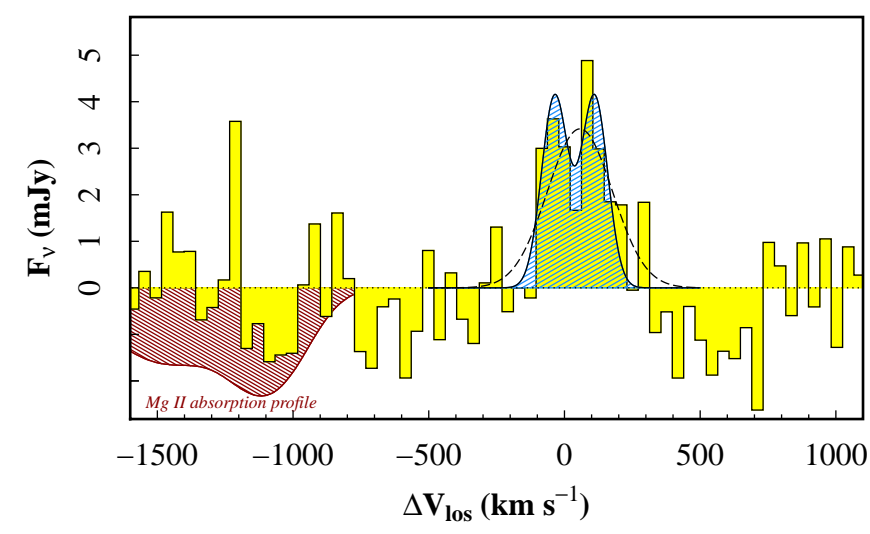

Figure 2. The $2 \mathrm{~mm}$ spectrum of SDSS J1506+54 obtained with IRAM PdBI, binned to a resolution of $40 \mathrm{~km} \mathrm{~s}^{-1}$. The channel noise (r.m.s.) is $1.1 \mathrm{mJy}$ per $40 \mathrm{~km} \mathrm{~s}^{-1}$ channel. We consider two model fits to the data: a single Gaussian profile, and a double Gaussian (§4). The latter is an approximation to the line profile of a rotating disc or ring, and the observed spectrum does show hints of a double-peaked profile. We note that there is a $3.2 \sigma$ spike in the spectrum close to the outflow velocity (as traced by blue-shifted $\mathrm{Mg}$ II absorption; we show the best-fit absorption profile of the Mg II doublet as a shaded region on an arbitrary flux scale). We acknowledge this is currently far too tentative to draw any conclusions, but we remark that - if real - this could be indicative of cold gas entrained in the outflow ( $(5.4)$.

even larger, if the mass distribution is as compact as suggested by the rest-frame UV morphology. There are two main possibilities: (i) the $r_{e}$ for the stellar mass is more widely distributed than $r_{e}$ of light measured in the F814W band (nearinfrared observations at the same resolution will be required to test this) such that the environment dominated by star formation is compact $\left(r \approx r_{e}\right.$ ), but molecular gas dominated, or (ii) the CO-emitting gas is more widely distributed than the compact optical emission. The lack of size and orientation constraints for the $\mathrm{CO}(2-1)$ emission makes any estimate of $M_{\text {dyn }}$ uncertain, however it would be unusual if the bulk of the gas reservoir (and presumably the concomitant dust) was beyond $r_{e}$ and configured in a way that did not completely obscure the UV-bright core.

\section{INTERPRETATION AND DISCUSSION}

\subsection{Comparison with other galaxies}

The measured $L_{\mathrm{IR}} / L_{\mathrm{CO}}^{\prime} \approx 1500$ is one of the highest measured for any star-forming galaxy (Fig. 3). Even if we accounted for an AGN contribution of $25 \%$ to our lowest estimate of $L_{\mathrm{IR}}$, SDSS J1506+54 is at the extreme tail of the $L_{\mathrm{IR}} / L_{\mathrm{CO}}^{\prime}$ distribution, with $L_{\mathrm{IR}} / L_{\mathrm{CO}}^{\prime} \approx 550$ in this case. There are a handful of other star-forming galaxies with similar $L_{\mathrm{IR}} / L_{\mathrm{CO}}^{\prime}$ properties; for example, the $z=0.575$ hyper-LIRG IRAS F00235+1024 (Combes et al. 2011) and the $z=0.633$ ULIRG IRAS F10398+3247 (Combes et al. 2012) both have $\mathrm{CO}$ line luminosities measured in the 2-1 transition, and are also at the extreme end of the $L_{\mathrm{IR}} / L_{\mathrm{CO}}^{\prime}$ distribution (Fig. 3). Several galaxies in the sample of Combes et al. (2012) have $L_{\mathrm{CO}}^{\prime}$ upper limits implying extreme $L_{\mathrm{IR}} / L_{\mathrm{CO}}^{\prime}$ and of these, two are obviously hosting AGN, as evident from their optical spectra.

Like SDSS J1506+54, F00235+1024 is not dominated by an AGN, although there is thought to be a non-negligible $(\approx 30 \%)$ AGN contribution to its total infrared emission (Verma et al. 2002, Farrah et al. 2002). F10398+3247 could

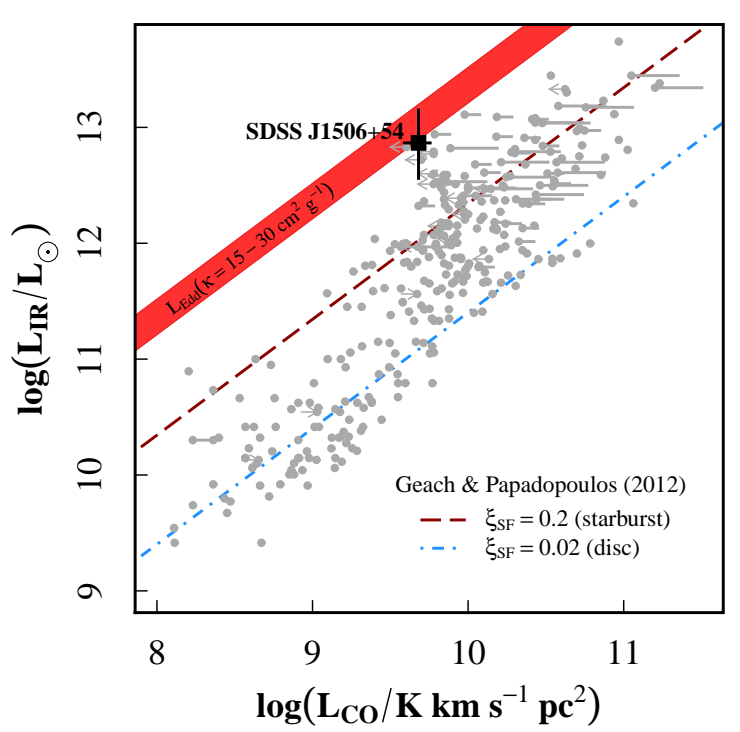

Figure 3. $L_{\mathrm{IR}}-L_{\mathrm{CO}}^{\prime}\left(J_{\mathrm{up}} \leq 3\right)$ plane for star-forming galaxies, showing the compilation of data for super star clusters, normal discs, local starburst galaxies, ULIRGs, submillimeter galaxies, hyper-LIRGs and circumnuclear starbursts from Andrews \& Thompson (2011). We plot $L_{\mathrm{CO}}^{\prime} J_{\mathrm{up}}>1$ uncorrected for sub-thermal excitation, but horizontal lines indicate how the points would move for corrections of $0.8 \leq r_{21} \leq 1$ and $0.5 \leq r_{31} \leq 1$ respectively. The shaded region shows $L_{\mathrm{IR}} \approx L_{\mathrm{Edd}}$ for optically thick dust emission (see \$5.2). We also show predictions from Geach \& Papadopoulos (2012) which models the global gas reservoir as a combination of dense star-forming gas and a diffuse quiescent cold gas phase. SDSS J1506+54 is close to the Eddington limit, with only a handful of other galaxies exhibiting similar ratios (Combes et al. $2011 \& 2012$ ).

also contain a deeply buried AGN, as it has a deep silicate absorption feature $\left(\tau_{\mathrm{Si}}>3\right.$, Dartois \& Muñoz-Caro 2007), often associated with nuclei blanketed by a dense screen of carbonaceous and silicate grains. The key difference between these galaxies and SDSSJ1506+54 is in optical morphology: F00235+1024 and F10398+3247 are interacting systems, where multiple UV/optical clumps can be identified in HST optical and NIR imaging (Stanford et al. 2000, Farrah et al. 2002, Combes et al. 2012), thus it is unclear whether these galaxies support such extreme $\dot{\Sigma}_{\star}$.

\subsection{Eddington-limited star formation}

How do we interpret such extreme systems? Can $L_{\mathrm{IR}} / L_{\mathrm{CO}}^{\prime}$ be driven to such high values through star formation alone? In individual galaxies, isolated star-forming cores might be Eddington limited, which is to say $\dot{\Sigma}_{\star}$ is capped by radiation pressure from the recently formed $\mathrm{O}$ and $\mathrm{B}$ associations (e.g. Scoville 2004, Shirley et al. 2003). However, when taken as a whole, galaxies form stars at sub-Eddington rates due to intermittency - the fact that only a small fraction of the cold gas reservoir is actually forming stars (Downes \& Solomon 1998, Murray, Quataert \& Thompson 2005, Andrews \& Thompson 2011). Under certain conditions however, one could envision a scenario where the majority of the gas reservoir could be driven to form stars at the Eddington limit. SDSS J1506+54 is an excellent candidate for this phenomenon.

In the absence of nuclear heating, and assuming the model of momentum-driven feedback (e.g. Murray, Quataert \& Thompson 2005) the upper limit of $L_{\mathrm{IR}} / L_{\mathrm{CO}}^{\prime}$ for star-forming galaxies is set by the Eddington luminosity. For optically 
thick dust emission $\left(\tau_{100 \mu \mathrm{m}}>1\right)$ :

$$
L_{\mathrm{Edd}}=\frac{4 \pi G c}{\kappa} X_{\mathrm{CO}} L_{\mathrm{CO}}^{\prime}
$$

(Andrews \& Thompson 2011), where here we set $X_{\mathrm{CO}}=3$ (see $\S 5.3$ ) and $\kappa$ is the Rosseland-mean dust opacity. In Figure 3 we show the Eddington luminosity predicted for $\kappa=5$ $10 \mathrm{~cm}^{2} \mathrm{~g}^{-1}\left(f_{\mathrm{dg}} / f_{\mathrm{dg}}^{\mathrm{MW}}\right)$, where $f_{\mathrm{dg}}^{\mathrm{MW}}=1 / 150$ is the Milky Way dust-to-gas mass ratio (see Andrews \& Thompson 2011). We assume a dust-to-gas ratio of $f_{\mathrm{dg}} \approx 1 / 50$, appropriate for dusty star-forming galaxies (e.g. Kovács et al. 2006). Increasing either $f_{\mathrm{dg}}$ (as might be appropriate in highly obscured galaxies), or $\kappa$, or decreasing $X_{\mathrm{CO}}$, lowers $L_{\mathrm{Edd}}$ for a fixed $L_{\mathrm{CO}}^{\prime}$.

Our chosen values aim to be conservative in this respect, such that no star-forming galaxies can be described as superEddington, but we caution the reader that in this parameter space the Eddington limit is not expected to be a hard edge. A simple framework that explains the intrinsic range in $L_{\mathrm{IR}} / L_{\mathrm{CO}}^{\prime}$ is the model of Geach \& Papadopoulos (2012) that predicts the minimal dense gas mass for a given $L_{\mathrm{IR}}$, and a range of gas mass ratios $\xi_{\mathrm{SF}}=M_{\text {dense }} / M_{\text {total }}$. Typically, $\xi_{\mathrm{SF}} \approx 0.02$ for quiescent discs and $\xi_{\mathrm{SF}} \approx 0.2$ for ULIRGlike systems (Fig. 3). The Eddington limit in equation (1) is approached as $\xi_{\mathrm{SF}} \rightarrow 1$, indicating that the majority of the gas in SDSS J1506+54 is in a dense, active phase and the intermittency is close to unity.

\subsection{Estimating the gas mass}

Recent studies of the local (U)LIRG population where wellsampled $\mathrm{CO}$ and ${ }^{13} \mathrm{CO}$ ladders are available (Papadopoulos et al. 2012a) show that, when high-J CO or heavy rotor $(\mathrm{HCN}$, CS) line transitions are included in radiative transfer models, the $M_{\mathrm{H}_{2}} / L_{\mathrm{CO}}^{\prime}=X_{\mathrm{CO}}$ factor increases by a factor $\sim 3$ 10 compared to the widely applied $X_{\mathrm{CO}}=0.8$ conversion 8 usually adopted for ULIRG-like systems (Papadopoulos et al. $2012 \mathrm{~b}$ ). The explanation is that in the supersonic turbulent discs of ULIRGs, a significant fraction of the gas mass is expected to be in a dense $\left(n>10^{4} \mathrm{~cm}^{-3}\right)$ phase (Padoan \& Nordlund 2002, Papadopoulos et al. 2012, Papadopoulos \& Geach 2012). This is not well traced by single low-J CO tracers, despite the fact that these were originally used to calibrate the value of $M_{\mathrm{H}_{2}} / L_{\mathrm{CO}}^{\prime}$ in ULIRGs (Downes, Solomon \& Radford 1993, Solomon et al. 1997, Downes \& Solomon 1998).

Here we assume that the majority of the gas reservoir is indeed in a dense, turbulent phase. Setting $X_{\mathrm{CO}}=3$ and $r_{21}=0.8$ (Papadopoulos et al. 2012a, Geach \& Papadopoulos 2012), we estimate $M_{\mathrm{H}_{2}}=(1.9 \pm 0.3) \times 10^{10} M_{\odot}$. This is in agreement with the minimum dense gas mass expected if $L_{\mathrm{IR}}$ is powered by Eddington-limited star formation, since one expects a maximum $\epsilon_{\star}=L_{\mathrm{IR}} / M_{\text {dense }} \approx 500\left(L_{\odot} / M_{\odot}\right)$ (Scoville 2004, Thompson et al. 2005, Thompson 2009). The $\epsilon_{\star}$ expected in feedback models is indeed close to the value actually measured both for resolved star-forming cores in spiral discs and even entire starbursts (Scoville 2004, Shirley et al. 2003). Similar values of $\epsilon_{\star}$ are obtained when one considers the mass-to-light ratio of a deeply dust-enshrouded $\left(\tau_{\text {IR }}>1\right)$ zero-age main sequence (ZAMS) population, with $L_{\mathrm{ZAMS}} \approx L_{\mathrm{IR}}$ (Downes \& Solomon 1998). It is important

\footnotetext{
${ }^{8}$ we omit the units of $M_{\odot}\left(\mathrm{K} \mathrm{km} \mathrm{s}^{-1} \mathrm{pc}^{2}\right)^{-1}$
}

to note that, regardless of the exact gas mass calibration, assuming that the majority of the infrared emission originates from enshrouded star-forming regions, the empirical scalings in Figure 3 clearly indicates that SDSS J1506+54 is close to the Eddington limit.

\subsection{Hints of gas entrained in a wind}

The compact starburst in SDSS J1506+54 should be effective in the launching of a powerful momentum-driven galactic wind, powered by radiation pressure on dust grains intermingled with the ISM, and the ram-pressure of supernovae detonations (Murray, Quataert \& Thompson 2005). The strongly blue-shifted $\mathrm{Mg}$ II lines are excellent evidence that this is the case (Tremonti et al. 2007, DS12).

The $2 \mathrm{~mm}$ spectrum of SDSS J1506+54 exhibits a marginally significant $(3.2 \sigma)$ narrow $\left(\sigma<40 \mathrm{~km} \mathrm{~s}^{-1}\right)$ spike close to the outflow velocity traced by Mg II (no RFI or telluric contaminant is expected at this frequency). We can draw no conclusions on such a low-significance feature, but we remark that if cold gas has become swept-up in the outflow traced by Mg II absorption, and have survived shock-heating, then we might expect to detect entrained cold gas in this way.

\section{SDSS J1506+54 AS AN EDDINGTON-LIMITED STARBURST} INDUCED IN A LATE-STAGE MERGER

\subsection{Formation}

We speculate that SDSS J1506+54 is the remnant of a major merger. In this picture angular momentum losses caused by the dissipation of energy in shocks and gravitational torques cause the collapse of disc-gas to the central regions in each parent, and subsequently into a single merged core. This is a classic picture of galaxy growth that has been supported for many years by both theory, numerical simulations and observation (Toomre \& Toomre 1972, Sanders et al. 1988, Hernquist 1989, Barnes \& Hernquist 1991, Sanders \& Mirabel 1996, Schweizer et al. 1996, Mihos \& Hernquist 1996, Wuyts et al. 2010, Hopkins et al. 2012).

Tidal interactions in earlier stages of the merger might well be responsible for the faint extended (over several kpc) optical light (Figure 1, P. H. Sell et al. in prep). Episodes of massive star formation must have occurred in the recent past (0.5-1 Gyr), with the A stars formed in (or moved to) relatively unobscured regions, in order to produce strong $\mathrm{H} \delta$ absorption observed in the optical spectrum. Indeed, the presence of post-starburst spectral features is entirely consistent with the typical timescales for equal-mass mergers (Lotz et al. 2008).

\subsection{Fate}

In the absence of additional cooling of gas, SDSS J1506+54 must be close to the cessation of star formation. At the current rate the gas will be consumed within a few tens of Myr, increasing the stellar mass by just $\sim 15 \%$. This will place the galaxy in the exponential tail of the stellar mass function at $z \approx 0.5$ (Moustakas et al. 2013), but the current starburst contributes only a modest fraction of the total mass. Nevertheless, this small addition can have important consequences for the properties of the descendant (Robaina et al. 2009, Hopkins et al. 2010, 2012, Hopkins \& Hernquist 2010).

Signatures of compact Eddington-limited starburst episodes could be found in the 'fossil record' of local spheroids. Integral field observations of the cores of local elliptical galaxies reveal examples of compact $(\sim 100 \mathrm{pc})$, 'kinematically decoupled', relatively young ( $\lesssim 5 \mathrm{Gyr}$ ) stellar components in 
a subset of the population (e.g. McDermid et al. 2006). The origin of these structures could well be found in powerful, compact starbursts such as the one we present. Star formation driven at extremely high $\dot{\Sigma}_{\star}$ could also have a critical impact on the form of the stellar initial mass function, due to the corresponding high cosmic ray densities in such environments (Papadopoulos et al. 2011).

\section{SUMMARY}

We have presented observational evidence of what we term a 'redline' starburst: a galaxy forming stars close to the Eddington limit assuming current models of stellar feedback. The measured $L_{\mathrm{IR}} / L_{\mathrm{CO}}^{\prime} \sim 1500$ is one of the highest measured for any galaxy. Our conclusion is that the SDSS J1504+54 system is a final stage merger, undergoing a high intensity circumnuclear starburst. If we are witnessing the intense - but fleeting - closing stages of the assembly of a massive stellar bulge, SDSS J1506+54 represents a unique opportunity to study the physics associated with this important evolutionary phase, and the mechanics of star formation at its most extreme.

\section{ACKNOWLEDGEMENTS}

We thank the anonymous referee for a constructive report that improved this paper. We are also grateful to Brett Andrews, Padelis Papadopoulos, Nathan Bastian and Aday Robaina for helpful discussions and advice. J.E.G. is supported by a Banting Fellowship. A.M.D. acknowledges support from the Southern California Center for Galaxy Evolution. P.H.S. acknowledges support through the NASA grant HST-GO-12019. This work was also supported by Chandra grant \#tGO0-11135.

\section{REFERENCES}

Andrews B. H., \& Thompson T. A. 2011, ApJ, 727, 97

Barnes, J. E., Hernquist, L., 1991, ApJ, 370 L65

Chary, R., Elbaz, D., 2001, ApJ, 556, 562

Combes, F., García-Burillo, S., Braine, J., Schinnerer, E., Walter, F., Colina, L., 2011, A\&A, 528, 124

Combes, F., Garcia-Burillo, S., Braine, J., Schinnerer, E., Walter, F., Colina, L., 2012, A\&A, arXiv:1209.3665

Dartois, E., Muñoz-Caro, G. M., 2007, A\&A, 476, 1235

Dale, D. \& Helou, G. 2002, ApJ, 576, 159

Diamond-Stanic, A. M., Rieke, G. H., Rigby, J. R., 2009, ApJ, 698, 623

Diamond-Stanic, A. M., Moustakas, J., Tremonti, C. A., Coil, A. L., Hickox,

R. C., Robaina, A. R., Rudnick, G. H., Sell, P. H., 2012, ApJ, 755, L26

Downes D., \& Solomon P. M. 1998, ApJ, 507, 615

Farrah, D., Verma, A., Oliver, S., Rowan-Robinson, M., McMahon, R., 2002, MNRAS, 329, 605
Gandhi, P., Horst, H., Smette, A., Hönig, S., Comastri, A., Gilli, R., Vignali, C., Duschl, W., 2009, A\&A, 502, 457

Geach, J. E., Papadopoulos, P. P., 2012, ApJ, 757, 156

Gilli, R., Vignali, C., Mignoli, M., Iwasawa, K., Comastri, A., Zamorani, G., 2010, A\&A, 519, 92

Guilloteau, S., Lucas, R., 2000, Imaging at Radio through Submillimeter Wavelengths, ASP Conference Proceedings, Vol. 217, edited by J. G.

Mangum and S. J. E. Radford. Astronomical Society of the Pacific, p.299

Heckman, T. M., Ptak, A., Hornschemeier, A., Kauffmann, G., 2005, ApJ, 634,161

Hernquist, L, 1989, Nature, 340, 687

Hopkins, P. F., Murray, N., Quataert, E., Thompson, T. A., 2010, MNRAS, 401, L19

Hopkins, P. F., Hernquist, L., 2010, MNRAS, 402, 985

Hopkins, P. F., Cox, T. J., Hernquist, L., Narayanan, D., Hayward, C. C., Murray, N., 2012, arXiv:1206.0011

Irwin, M. J., Ibata, R. A., Lewis, G. F., Totten, E. J., 1998, ApJ, 505, 529

Kennicutt, R. C. Jr., 1998, ARA\&A, 36, 189

Kovács, A., Chapman, S. C., Dowell, C. D., Blain, A. W., Ivison, R. J., Smail, I., Phillips, T. G., 2006, ApJ, 650, 592

Lotz, J. M., Jonsson, P., Cox, T. J., Primack, J. R., 2008, MNRAS, 391, 1137

McDermid, R. M., et al., 2006 MNRAS, 373, 906

Mihos, J. C., Hernquist, L., 1996, ApJ, 464, 641

Murray, N., Quataert, E., Thompson, T. A., 2005, ApJ, 618, 569

Moustakas, J., et al., 2013, arXiv:1301.1688

Narayanan, D., Hayward, C. C., Cox, T. J., Hernquist, L., Jonsson, P., Younger, J. D., Groves, B., 2010, MNRAS, 401, 1613

Padoan P. \& Nordlund 2002, ApJ, 576, 870

Papadopoulos, P. P., Thi, W.-F., Miniati, F., Viti, S., 2011, MNRAS, 414, 1705

Papadopoulos, P. P., van der Werf, P. P., Xilouris, E. M., Isaak, K. G., Gao, Y., Mühle, S., 2012, MNRAS, 426, 2601

Papadopoulos, P. P., van der Werf, P., Xilouris, E., Isaak, K. G., Gao, Y., 2012, ApJ, 751, 10

Papadopoulos, P. P., Geach, J. E., 2012, ApJ, 757, 157

Rieke, G. H., Alonso-Herrero, A., Weiner, B. J., Pérez-González, P. G.,

Blaylock, M., Donley, J. L., Marcillac, D., 2009, ApJ, 692, 556

Robaina, A. R., et al., 2009, ApJ, 704, 324

Sanders, D. B., Soifer, B. T., Scoville, N. Z., 1988, Science, 239, 625

Sanders, D. B., Mirabel, I. F., 1996, ARA\&A, 34, 749

Schweizer, F., 1996, AJ, 111, 109

Scoville N. Z. 2004, The Neutral ISM in Starburst Galaxies, Astronomical Society of the Pacific Conference Series, 320, p.253

Shirley, Y. L., Evans, N. J., II, Young, K. E., Knez, C., Jaffe, D. T., 2003, ApJS, 149, 375

Solomon P. M., Downes D., Radford S. J. E., \& Barrett J. W. 1997, ApJ, 478, 144

Solomon P. M., Vanden Bout, P. A. 2005, ARA\&A, 43, 677

Stanford, S. A., Stern, D., van Breugel, W., De Breuck, C., 2000, ApJS, 131, 185

Thompson T. A., Quataert E., \& Murray N. 2005, ApJ, 630, 167

Thompson T. A. 2009, The Starburst-AGN connection, Astronomical Society of the Pacific Conference Series, 408, p.128

Toomre, A., Toomre, J., 1972, ApJ, 178, 623

Tremonti, C. A., Moustakas, J., Diamond-Stanic, A. M., 2007, ApJ, 663, L77

Verma, A., Rowan-Robinson, M., McMahon, R., Efstathiou, A., 2002, MNRAS, 335, 574

Wuyts, S., Cox, T. J., Hayward, C. C., Franx, M., Hernquist, L., Hopkins, P. F., Jonsson, P., van Dokkum, P. G., 2010, ApJ, 722, 1666 